\title{
The interaction of perceptual processes and ambiguous sentences*
}

\author{
T. G. BEVER \\ Columbia L'nilersity. New York. New York 1002\% \\ M. F. GARRETT \\ Massachusetts Institute of Technolugy', Cambridge, Massachusetts 02139 \\ and \\ R. HURTIG† \\ New York University', New York, New York 10003
}

\begin{abstract}
Cincrai principles of spech perception resolve several experimental conflicts about whe ther listeners interpret one or all meanings of an ambiguous sentence. We arguc that during an ambiguous clause, both meanings are processed, but immediately after the clatuse is over, it is recoded with only one meaning retained. This model resolves the apparently conflicting results of previous experiments; it also predicts that underlying structure anbiguity in incomplete clauses increases comprehension time. In complete clauses, ambiguity does not increase relative comprehension time; it may redice comprehension time for ambiguities whose interpretations are perceptually distinct in those tasks where either meaning is appropriate. Two new experiments offer pretiminary confirmation of these predictions
\end{abstract}

Many sentences could mean more than one thing, but in a normal context we usually notice only one meaning. This fact presents a puzzle to the student of language behavior: do listeners unconsciously decide between the interpretations of an ambiguous sentence or do they analyze the first meaning that comes to mind without analyzing the other? Some recent experimental studies have presented evidence that listeners unconsciously anlalyze both meanings of an ambiguous sentence, even though they are aware of only one meaning. Other studies have supported the upposite claim, that listeners ordinarily analyze only one meaning of an ambiguous sentence. In this paper, we review a theory of the nomal mechanisms of speech processing, use this theory to resolve the previous conflicting results. and present two experimental results which support our view of the pereeption of ambiguous sequences.

Recent research has suggested three principal features of speceh perception: (I) the clause is a primary perceptual unit. (2) within each clause direct mapping rules assign underlying structure relations among lexical items, and (3) after cach clause is processed it is lecoded into a relatively abstract form, thereby leaving immediate storage available for processing the next clause

*Research was supported by PHS Grant GM 16735 to the Rockefeller University, Grant 5 RO1 HD05168 from the National Institute of Child Health and Human Development to the Massachusetts Institute of Technology. and Office of Iiducation Grant OH(-(-72-1403. Reprint requests should be sent to T. (i. Hever. Department of Psychology. Columbia University. New York. New York 10027. We are indebted to ldward Walker, Iavid Hakes. and Robert ( hiarello for advice on the mantuscript.

$\$$ Now at Columbia C'niversity.
Responses to nonspeech stimuli presented during sentences indicate that closure occurs at clause boundaries. Clicks are mislocated as occurring at such points (Fodor \& Bever, 1965; Garrett, 1965; Garrett et al, 1966), but not at points of low transitional probability in general (Bever, Lackner, \& Stolz, 1969). nor at all surface phrase structure breaks in general (Bever, Kirk, \& Lackner, 1969). If speech is switched from one ear to the other, the point of switching is most accurately located if it occurs at a clause boundary (Wingficld \& Klein, 1970). Reaction time to clicks between clauses is faster than to clicks not between clauses when Ss are motivated to emphasize sentence perception (Holmes \& Forster, 1972: Abrams, 1973). These studies and others demonstrate the importance of the clause as a processing unit. ${ }^{1}$

The second principle is that within each perceptual unit, the underlying structure relations are assigned by direct projection from the lexical sequences. A set of perceptual mapping rules are sensitive to specific features of the surface sequence that signal particular underlying structure relations. Sentences with explicit relative clause markers (e.g., "who," "when") are less complex than those without (Fodor \& Garrett. 1967: Hakes \& Cairns, 1970). Similarly, explicit marking of complement constructions (e.g., presence of "that") is facilitating (Hakes, 1971). Sentences with verbs having more potential underlying structure roles are also relatively complex (Fodor, Garrett, \& Bever. 1968 Hakes, 1971: Holmes \& Forster, 1972). This (i)llows from the fact that such verbs give less specific cues 11 the underlying structure of the sentences they are in.

Both the clause segmentation and internal labeling processes place heavy emphasis on information inherent to individual lexical items as well as surface properties of sentences. On this view, sentence comprehensiom is the 
joint product of the projection of potentia: clauses and the analysis of underlying grammatical relations within each clause (for reviews, see Bever, 1970: Fodor, Bever, $\&$ Garrett. in press). On this view, the surface constituents are directly marked for their primary underlying structure relations, e.g.. "actor, action, object, modifier."

The perceptual mapping operations do not apply homogeneously during a sentence. Accordingly. changes in sentence processing activity are reflected in variations in attention to nonspeech stimuli (e.g., the click location and reaction time studies already referred to). Abrams and Bever (1969), Valian (1971), and Seitz (1972) have argued that maximum processing load occurs at the end of clauses. Abrams and Bever interpret the phenomena in the following way: during a clause, listeners accumulate information and hypotheses concerning its potential underlying structure(s): the end of the clause is the point at which a particular complete underlying structure is determined. More recent research has suggested that once underlying structure of a clause is determined. its initial phonetic representation is removed from immediate storage. While immediate recall of the meaning of the first clause of a two-clause sentence is virtually perfect, free recall of the exact words in that clause is poor (Jarvella, 1970; Jarvella \& Herman, 1972). Consistent with this is the fact that latencies to identify a probe word are faster if the word is drawn from the terminal clause of a sentence than if drawn from an immediately preceding ciause (Ćapian, 1972). (In all these studies, simple serial position effects were controlled for.)

The following picture of speech perception emerges from these studies. During a clause a listener projects potential organizations for the underlying semantic relations in it. At the end of each clause a structure is assigned and the external form of the clause itself is dropped from immediate memory. This sets the scene for the empirical question in the present paper: How does such a perceptual mechanism for speech deal with sentence ambiguity? Specifically, do listeners compute all the meanings of an ambiguous sequence or just one? We shall argue that within a clause, more than one potential underlying structure may be simultaneously' processed. but at the end of the clause, one structure is chosen and the others are dropped. If the plausible structural alternatives are analyzed during a clause, then an ambiguous sequence should require extra processing space during the clause containing it. After the clause is recoded with a single interpretation, a subsequent task should not reflect the effect of an original ambiguity. This view explains the differences in the outcome of previous studies, although none were conducted specifically to test it.

Consider the experimental support for the view that both meanings of an ambiguous sequence are available within a clause. Foss (1970) found that discrimination reaction time is slower immediately following an ambiguous portion of a sentence than following a corresponding nonambiguous portion. Lackner and Garrett (1972) showed that the interpretation of an ambiguous sentence is affected by a simu!taneously presented disambiguating context. They conclude from this that both interpretations of an ambiguous sentence are immediately available during its processing. MacKay (1966) found that the time to complete an ambiguous sentence fragment [e.g., (1)] is longer than the time to complete a corresponding fragment which is similar but not ambiguous [e.g., (2)].

(1) After the right turn was...

(2) After the left turn was ...

Finally, Mistler-Lachman (1972) reports that visually presented ambiguous stimulus sentences occasion relatively long times to think up a following "appropriate" sentence. Her technique encouraged Ss to start their responses as soon as possible, and her responses (measured from stimulus onset) were very short (ca. $2 \mathrm{sec}$ ). It is likely that her Ss started thinking about (and sometimes even uttering) their response before they had finished interpreting the stimuli. Thus, her results are consistent with the view that ambiguity complicates tasks that are initiated before sentence interpretation is completed.

By contrast, a number of other experiments are consistent with the view that postsentence tasks do not reveai any added complexity as a lunction of an ambiguity within the sentence. Foss, Bever, and Silver (1968) found evidence that after a sentence interpretation is completed, only one meaning is available to the listener. They followed an ambiguous sentence with a picture appropriate either to the more frequent interpretation of the ambiguous sentence or to the less frequent interpretation. S's latency to respond was longer when the picture required the less frequent interpretation of the ambiguous sentence. Foss et al conclude that their results "support a model of normal sentence comprehension which states that Ss typically assign only one immediate interpretation to an ambiguous sentence. Only if that interpretation is found to be incorrect does $S$ reinterpret the sentence [Foss et al. 1968. p. 306]."

Carey, Mehler, and Bever (1970) used the same experimental paradigm with sentences and pictures as did Foss et al. They preset Ss to expect a sentence with one of the two phrase structures illustrated in (3) and (4). Then $S$ heard an ambiguous sentence like (5) followed by a picture that could be appropriate or not to both interpretations.

(3) They are (smiling men)

(4) They (are fixing) lunches

(5) They are lecturing doctors

They found that the "unseen" meaning of the critical 
sentence affected S's reaction time only if $S$ later reported having noticed the ambiguity at the time. That is, the two meanings of an ambiguous sentence interacted only if they were both noticed consciously. If the ambiguity was unnoticed, the sentences were responded to as though unambiguous.

Though the two sets of experiments we have briefly described lead to different conclusions about the effects of ambiguity on sentence comprehension, the conflicting results are interpretable in terms of differences in the experimental paradigms used. Those experiments which show an effect of ambiguity used tasks initiated during the processing of the sentence, while those showing no effect used tasks initiated after processing of the sentence was presumably complete. In the context of the perceptual theory discussed above, the end-of-sentence effects in these experiments may be viewed as a special case of clause boundary effects: tasks that occur before such boundaries are complicated by the presence of both interpretations of ambiguities, while subsequent tasks are not.

We report two experiments below in which we examine this possibility and related issues. We use two "sentence completion paradigms," one applied to the "postsentence" case and one applied to the "within-sentence" case.

\section{EXPERIMENT I}

Foss et al's and Carey et al's evidence support the claim that one meaning for a sentence is chosen at its conclusion. We ran a study comparing the time to process ambiguous and nonambiguous sentences with a technique different from that used by Foss et al and Carey et al but still focused on the interval immediately following the presentation of an ambiguous sentence. The dependent variable was the time taken to think up the next sentence of a hypothetical story. This was similar to Mistler-Lachman's (1972) response requirements. However, the materials were designed to have only one-third of the stimulus sentences to be ambiguous for each $\mathrm{S}$ (in her study, the proportion of ambiguous sentences was three-fourths): this was to reduce the likelihood that $S$ would notice the ambiguities. Also, $\mathrm{S}$ was explicitly instructed to interpret each sentence before thinking up a response, and his reading light was turned off when he started talking: these measures were to reduce the possibility that $S$ would start the response task before completing the interpretation of the stimulus.

\section{Method}

\section{Materials}

Twenty-seven 7- to 9-word ambiguous sentences were constructed. Three types of ambiguity were used (nine sentences of each type): lexical ambiguities. in which there are two distinct meanings for one of the words in the sentence. s.e. (6il): surfiace phrase structure ambiguities, in which there are two bracketings of the words in the sentence, e.g., (7a); and underlying structure ambiguities, in which the two readings of the sentences are reflected only in different underlying "logical" relations among the words, e.g., (8a) [see MacKay \& Bever (1967) for a description and further examples of these distinctions]. Corresponding to each interpretation for an ambiguous sentence there was a nonambiguous sentence which differed from it in one word and which presented unambiguously one or the other of the structures of the ambiguous version [e.g. the $b$ and $c$ versions of $(6),(7)$, and (8)] .

6 (a) Be sure that you take the right turn

(b) Be sure that you take the left turn

(c) Be sure that you take the correct turn

7 (a) The paper presented carefully limited analyses of the problem

(b) The paper presented today limited analyses of the problem

(c) The paper presented very limited analyses of the problem

8 (a) The shooting of the Indians bothered the agent

(b) The shooting from the Indians bothered the agent

(c) The shooting at the Indians bothered the agent

These materials were arranged into three experimental groups of 27 sentences, such that each group contained 3 ambiguous sentences of each type as well as 6 nonambiguous sentences of each type. In this way, each sentence that was ambiguous in one group had one of its nonambiguous versions in a second group and its other nonambiguous version in the third group. Each sentence was typed without punctuation on a $3 \times 5$ in. card.

\section{Subjects}

The Ss were 18 male Harvard summer school students (all native speakers of English) who volunteered for the experiment and were paid for their participation.

\section{Procedure}

Each $S$ was tested individually with a screen separating him from $E$. The stimulus sentences were presented on cards one by one through a slot in the screen. $E$ instructed $S$ first to read each sentence and then to think up "the next sentence of a story in which the stimulus sentence might occur." S pressed a switch as soon as he started to respond. which turned off his reading light. A timer recorded the interval between the initial presentation of a sentence and S's movement of the switch. All Ss were given the same 6 practice sentences before the experimental set of 27 sentences was presented. Each response was categorized as corresponding to one of the two interpretations of the stimulus ambiguity. There were two indicants of which interpretation the $S$ intended: inspection of S'S responses by $E$. and posttest questioning of $\mathrm{S}$. To obtain the second measure. each $\mathrm{S}$ was played his original tape-recorded responses and after each sentence was asked if he had noticed the ambiguity of the stimulus sentence. Regardless of his answer to this question. the nature of the ambiguity was explained and he was asked which of the two meanings he had in mind when he had produced his response during the experimental session. There were no disagreentents between E's scoring and Ss' reports: when the story continuation did not indicate clearly to $E$ the meaning $S$ had in mind. S's posttest response was used to assign the continuation to one of the two interpretations. [In a few cases (less than 2r). Ss claimed to have noticed the ambiguity and to have had both meanings in mind or some third interpretation when they produced their respone: these responses wat siminated from our analy sw. 
Table 1

Time (Seconds) to Start the Responses to Ambiguous and Unambiguous Sentences in Experiment I

\begin{tabular}{|c|c|c|c|}
\hline & \multicolumn{3}{|c|}{ Type of Ambiguity } \\
\hline & Lexical & $\begin{array}{l}\text { Surface } \\
\text { Structure }\end{array}$ & $\begin{array}{l}\text { Underlying } \\
\text { Structure }\end{array}$ \\
\hline Ambiguous sentences & 5.28 & 9.03 & 8.83 \\
\hline Corresponding unambiguous sentences & 4.93 & 9.40 & 9.72 \\
\hline Mean difference of responses (ambiguous-unambiguous) & +0.35 & -0.27 & -0.89 \\
\hline Percentage of Ss responding faster to ambiguous versions & $34 \%$ & $56 \%$ & $82 \%$ \\
\hline
\end{tabular}

\section{Results}

The time to start the response sequence was about $8 \mathrm{sec}$ (note that this includes reading time for the stimuli). Overall, lexical sentences (both ambiguous and unambiguous) were responded to more quickly than were sentences of other types $(p<.01$ by two-tailed sign test across Ss for ambiguous and nonambiguous sentences separately). Other differences between types of sentences were not significant. The results in Table 1 show an interaction of the effect of ambiguity with ambiguity type. For lexical and surface structure ambiguities, there was no significant difference between ambiguous sentences and their nonambiguous controls (tested by sign test across Ss and across sentences); for underlying structure ambiguities, the mean for ambiguous sentences was significantly shorter than the mean for the control sentences ( $p<.03$, two-tailed sign test across Ss; $p<.05$ across sentences). The overall pattern that underlying structure ambiguities facilitated latencies compared with their controls more than the other ambiguity types was significant $(p<.01$ by two-tailed sign test across Ss): the fact that the lexical structure ambiguities slowed latencies compared with their controls more than the other ambiguity types was also significant $(\mathrm{p}<.01$ by two-tailed sign test across Ss).

This relative speed of underlying structure ambiguities appears to be due almost entirely to a decrease in the time Ss take to arrive at a meaning corresponding to that of the slower of the two unambiguous versions. We compared separately the mean response time for each interpretation of an underlying structure ambiguity with its corresponding unambiguous control. The response times of ambiguous interpretations corresponding to the slower of the two controls were significantly faster than controls $(p<.05$ by two-tailed sign test across sentences): the response times of the ambiguous interpretations corresponding to the faster of the two controls were not significantly faster than their controls. That is, the relative speed of processing the underlying structure ambiguities is due primarily to dropping some of the responses to the slower interpretation rather than to increasing the speed of the responses to the faster interpretation.

\section{Discussion}

The finding that ambiguous and unambiguous "lexical" sentences are responded to faster than are other types may be due to the relative simplicity of the constructions used in the lexical sentences: the potential presence of structural ambiguity necessitates a relatively complex construction even in the nonambiguous versions [cf. the difference between $(6 b)$ and $(6 c),(7 b)$ and $(7 c)$, and $(8 b)$ and $(8 c)]$.

However, our careful matching of ambiguous and unambiguous stimuli allowed us to examine the results for the effects of ambiguity, despite the overall differences. This comparison shows that there is no increase in story-continuation time as a consequence of ambiguity in a stimulus sentence (numerically it is, in fact, a decrease). This is compatible with those previous experiments that showed that ambiguity does not complicate processing once a stimulus sentence is finished. That is, when $S$ is encouraged to complete the interpretation of a sentence before thinking up a response, there is no interference from the "unselected" irreaning.

An unexpected result was the fact that sentences with underlying structure ambiguities were responded to faster than their unambiguous controls. This result is, however, entirely compatible with the view that Ss were computing both interpretations independently during the stimulus presentation. Since either interpretation was acceptable in the experimental situation, "savings" in response times could result [given that the first interpretation to be consciously perceived stops all further sentence processing (e.g., Smith, 1968)]. Suppose that we view the perception of each meaning of an underlying structure ambiguity as an independent event with a range of times for its completion. When the structures are combined within an ambiguous stimulus sentence, the probability that at least one of the meanings is perceived by a particular time is, in general, greater than the probability for either of the interpretations in isolation, viz, (9).

$$
\begin{aligned}
\text { (9) } P_{\left(1 \text { or 2) before } t_{j}\right.}= & P_{1}+P_{2}-P_{1} P_{2} \\
P_{1}= & \text { probability that mean- } \\
& \begin{aligned}
\text { ing } 1 \text { is perceived by } \\
\text { a particular time, } t_{i}
\end{aligned}
\end{aligned}
$$

$P_{2}=$ probability that mean . ing 2 is perceived by a particular time. $t_{i}$ 
$P_{11}$ or 2) is higher than $P_{1}$ or $P_{2}$ alone if both are between 1 and 0 .

A physical analogue to the comprehension of ambiguous sentences can clarify the application of Eq. 9 to the results in Experiment I. Suppose we presented a blindfolded $S$ with a box containing one tennis ball. The box has a hole in the top of it large enough to allow the $S$ to bring out the ball. The $S$ is asked to reach into the box and bring out a ball as quickly as he can. Clearly, if there are in fact two tennis balls in the box, tennis ball discovery time would be shorter than if the box contains only one ball, if it does not matter which of the two the $S$ comes up with. Equation 9 expresses exactly the "savings" that would result (in this case, "perception time" corresponds to "tennis ball discovery time").

The times to find each ball alone would have a distribution range. Suppose that the slowest response to one of the balls was intrinsically faster than to the other, as could happen if one were easier to grasp. This would have the effect that the slow responses to the harder ball that occur when it is alone would never occur when it is together with the faster ball. This is because even the slowest response to the easier ball would occur before some of the responses to the harder one. Finally, if the two balls are not independent of each other (e.g., if they are attached), this would reduce the effect of Eq.9, since finding one ball would be tantamount to finding the other.

It would be simplistic to apply this physical model directly to sentence perception. However, it does exemplify the possible interactions between the meanings of an ambiguity. In the ambiguous version, the mean time to perceive the structure corresponding to that of the slower control sentence should be affected more than the time to perceive the structure corresponding to the faster control sentence. This follows from the fact that the probability of the faster interpretation reaches 1 before the probability of the slower meaning (by definition, if the response distributions to the two meanings have the same shape). Accordingly, the mean time of those responses associated with the slower structure which do occur will tend to be from the faster part of its distribution. This was exactly the result found. (Notice that there may be an interaction between the probability of a meaning and its relative time of computation; but we have no independent assessment of the probabilities for the separate meanings in our stimulus sentences.)

Previous experiments have brought out a variety of behavioral differences in response to the different structura! types of ambiguity. For example, in the sentence completion experiment (MacKay, 1966), it was found that ambiguity at the underlying structure level had the largest rerarding effect on completion time for sentence fragments. Yet it is these ambiguities in Experiment I which were responded to significantly faster than their corresponding controls. This apparent inconsistency can be resolved in terms of the perceptual model we sutlined above.

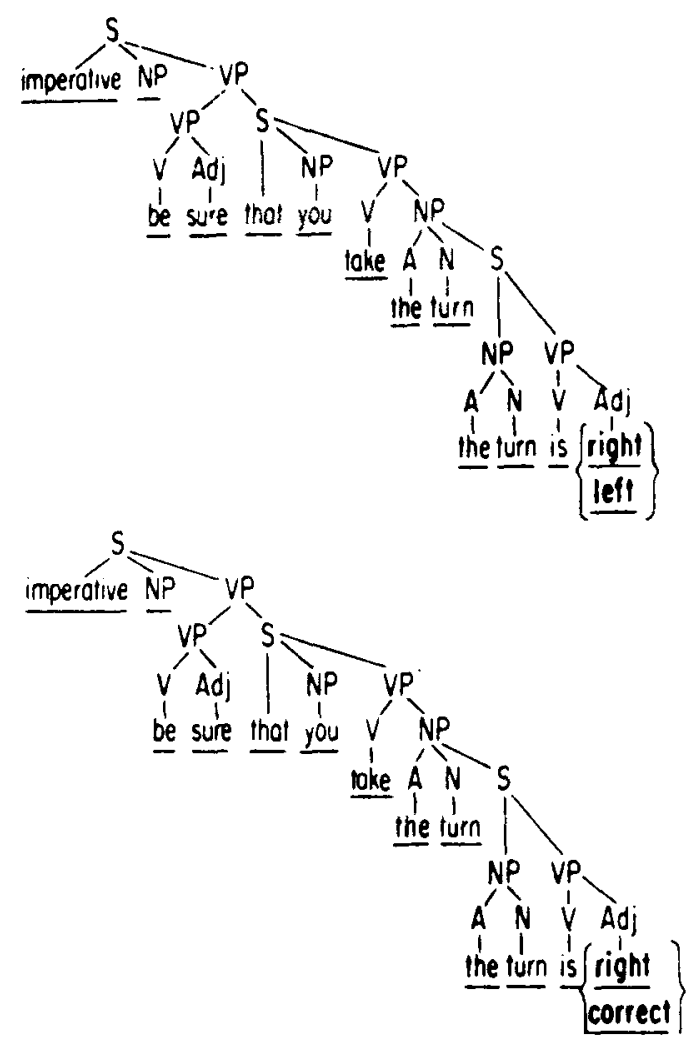

Fig. I. Lexical structure ambiguity.
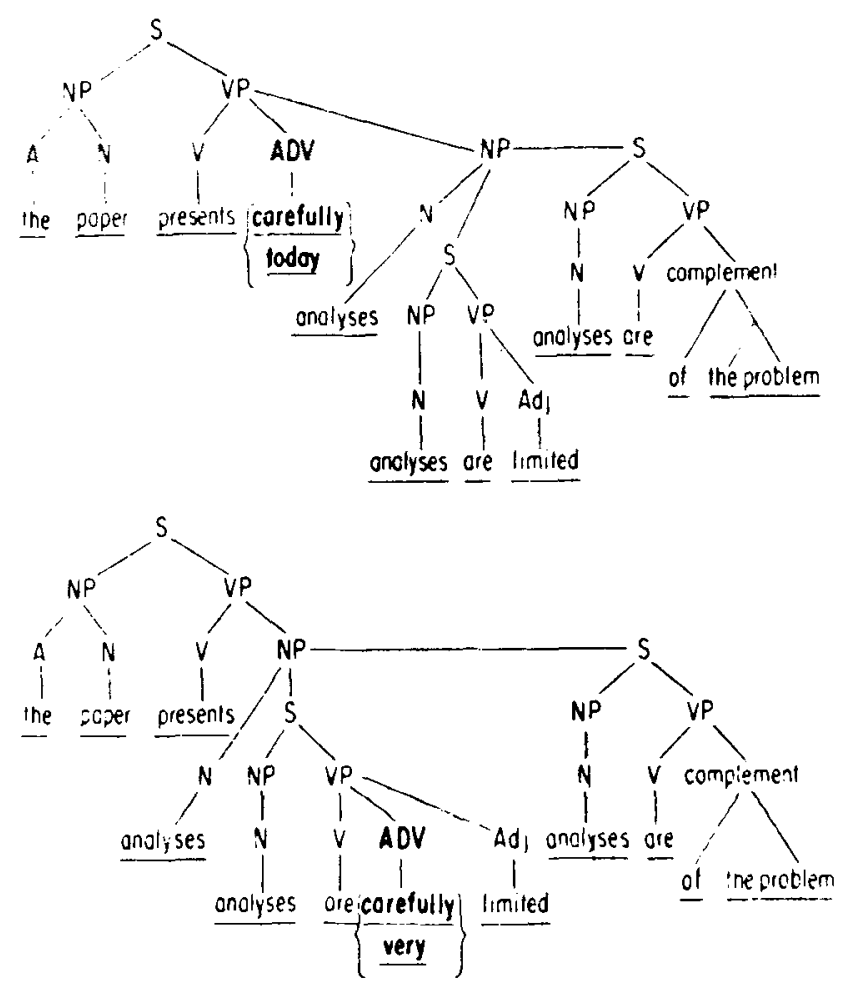

Fig. 2. Surface structure ambiguity. 

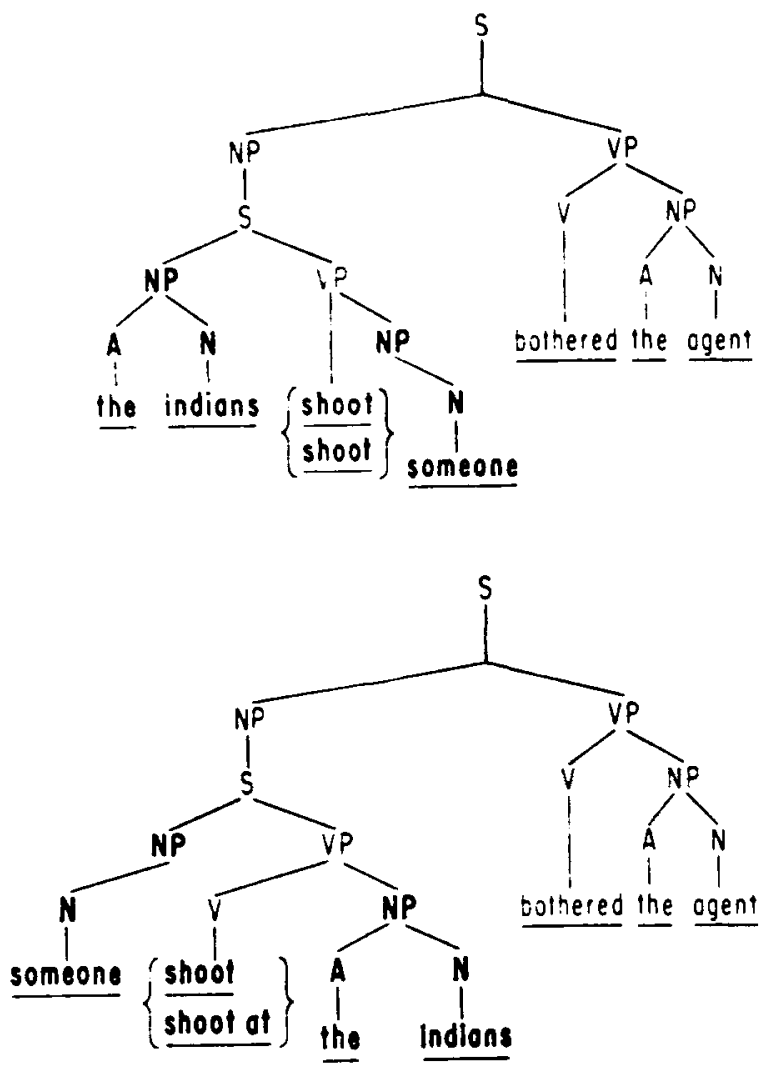

Fig. 3. Deep structure ambiguity.

Consider first the relative independence of the perceptual mapping rules used to analyze the two structural interpretations in each kind of ambiguity. Underlying structures are shown in Figs. 1.3 for the sample ambiguities (6). (7), and (8). The two interpretations of lexical ambiguities have identical underlying structures; the structures for the two interpretations of surface structure ambiguities have a minor difference, characteristically in the placement of a modifier: and finally, the structures for the two interpretations of underlying structure ambiguities are most different. characteristically in the assignment of several of the basic grammatical relations, "agent, action, object, modifier." In the perceptual model outlined above, direct perceptual operations map surface sequences onto underlying relations. Thus, the types of ambiguities differ in the extent to which the perceptual mapping operations used to perceive one interpretation are identical with those used to perceive the other interpretation. In lexical ambiguities, the perceptual rules for the two meanings are virtually identical; in surface structure ambiguities, they may differ slightly; and in underlying structure ambiguities they differ considerably. Initial experimental support for the view that the meanings of underlying structure ambiguities are relatively independent comes from the findings of MacKay and Bever (1967). They reported that the time to discover an underlying structure ambiguity is strictly an inverse function of the relative probability of the second meaning that $S$ perceives: this is just what would be expected if the meanings are perceptually independent. The relation found for the other two ambiguity types was not of this form, suggesting that the two meanings in those ambiguity types are not perceptually independent (cf. MacKay \& Bever, 1967, p. 195, Fig. 2).

The experimental differences between the different kinds of ambiguities found in tasks involving sentence comprehension may be explained by the relative perceptual independence of the interpretations in each ambiguity type. In a task like Experiment $I$ in which either interpretation is appropriate and the response task comes after the sentence boundary, independence of the two meanings in an ambiguity is an advantage: it leads to faster responses as predicted in (9). Accordingly, the classification of ambiguities according to the relative independence of their interpretations predicts that underlying structure ambiguities would show greatest facilitation over unambiguous controls, with surface structure ambiguities showing some relative facilitation and lexical ambiguities showing relatively little: this was the obtained result.

This perceptual differentiation of ambiguity types may account for the contrary effects of underlying structure ambiguities when incomplete sentences are the stimuli (MacKay, 1966): in these paradigms, the presence of an underlying structure ambiguity retarded the response compared with its unambiguous control. According to our perceptual theory, $\mathrm{S}$ is carrying out two distinct perceptual operations during presentation of a sentence fragment with an underlying structure ambiguity which yield two potential underlying structures for the lexical sequence. If called upon to complete the sentence fragment before the clause boundary, $\mathrm{S}$ must choose prematurely between the two incomplete and independent interpretations of the sentence in order to find a semantic basis for a completion. It is this active, premature choice which increases the time taken to complete an underlying structure ambiguous sentence fragment. Surface structure ambiguities have less effect on completion time because the perceptual operations involved in the two interpretations are similar to each other, thereby reducing the effect of choosing one or the other. Lexical ambiguities have the least effect in the sentence fragment completion paradigm because their interpretations use identical perceptual mapping operations and no active choice of competing perceptual operations is required.

The principle of recoding at clause boundaries outlined above resolves another apparent contradiction in previous studies of ambiguity. While MacKay (1966) found that lexical ambiguities have the least effect on sentence completion. Foss et al (1969) and Cairns (in press) found that such ambiguities are more difficult for $\mathrm{S}$ to recode into a second interpretation after having assigned 
Table 2

Mean Completion Time (Seconds) as a Function of Clause Structure of Fragment to $\mathrm{Ss}^{*}$

\begin{tabular}{lcc}
\hline \multicolumn{1}{c}{ Type } & $\begin{array}{c}\text { Incomplete } \\
\text { Clause } \dagger\end{array}$ & $\begin{array}{c}\text { Complete } \\
\text { Clause }{ }^{\dagger} \dagger\end{array}$ \\
\hline $\begin{array}{l}\text { Ambiguous } \\
\text { Fragments }\end{array}$ & 6.53 & 6.30 \\
$\begin{array}{l}\text { Nonambiguous } \\
\text { Fragments }\end{array}$ & 6.21 & 6.24 \\
Difference & 0.32 & 0.06 \\
\hline
\end{tabular}

*Original data taken from MacKay (1966), Appendix A, excluding "multiple ambiguities."

$t_{N}=20$, lexical $=7$, surface $=7$, deep $=6$

t†N $=22$, lexical $=7$, surface $=7$, deep $=8$

it one interpretation. That is, it is apparently easier to reinterpret a sentence with a deep structure ambiguity than a sentence with a lexical ambiguity. The assumption that lexical material is dismissed from immediate storage after a clause boundary offers one reason why this should be so: just after an ambiguous sentence is completed, the listener has dismissed the lexical material but still has available an underlying interpretation of the major phrases and their semantic relations. Reorganizing these relations may be easier than recovering the discarded lexical material in order to find a different interpretation for a particular word.

\section{EXPERIMENT II}

We have suggested that the point in a sentence at which $S$ starts to organize the response governs the effect of stimulus ambiguity on performance: if $S$ must respond at a point before perceptual closure, then ambiguity increases processing time; if the response task occurs after perceptual closure, then the ambiguity has no retarding effect unless the experimental situation demands an.interpretation different from the one $\mathrm{S}$ has already selected. In a situation like Experiment $I$ in which either interpretation of a sentence is acceptable, ambiguity in a complete unit can result in reduced processing time if the two meanings are perceptually distinct.

This interpretation of Experiment I depends on the assumption that Ss followed our instructions and did not start preparing their responses until after reading (and comprehending) each stimulus sentence. The response times were longer than those of Mistler-Lachman (1972), suggesting that our instructions were successful. However, a stronger test of the hypothesis that ambiguity increases complexity before perceptual clause closure and may decrease it thereafter would be to vary the stimuli themselves rather than the instructions: that is, a more critical test would be a direct comparison of the effect of ambiguity in response to speech stimuli that are themselves complete or incomplete perceptual units. MacKay (1966) presents part of his completion data by individual sentence fragment, allowing comparison of responses to stimuli that were complete perceptual units with those that were not. We categoried his stimulus sentence fragments into two types, those which could be complete clauses at the underlying structure level [e.g., (10) and (11)] and those that were incomplete [e.g., (12) and (13)]. Table 2 presents the completion times for the different types of ambiguous fragments and their unambiguous controls [from MacKay (1966), appendix] .

(10) Although I knew the new position had advantages ...

(11) Brahms, knowing how great symphonies sound, played ...

(12) After her injury that summer she couldn't bear ...

(13) Even if I did laugh at the church, I . .

The fragments which were complete clauses in themselves show no significant effect of the ambiguity: fragments that could not be interpreted as complete clauses do show such an effect. Thus, MacKay's data also offer confirmation of our general view of the relation between clause completion and sentence ambiguity.

With a modification of the sentence completion paradigm, we examined further the hypothesis that ambiguity interacts with perceptual closure. In this experiment, Ss responded to incomplete sentence fragments which were varied so as to be either complete clauses or incomplete clauses. The results show that ambiguity of a sentence fragment increases complexity of processing primarily when the fragment is itself an incomplete clause.

\section{Method}

\section{Materials}

Six incomplete sentence fragments 5 to 12 words in length of each type of ambiguity were adapted from MacKay (1966) [e.g., (14a-c), (15a-c), and (16a-c)]. Each fragment had three ambiguous versions and three nonambiguous versions: in one ambiguous version the fragment was an incomplete clause (a), in a second version it was a complete clause (b), and in the third version it was a complete clause with the addition of the first word of a second clause (c). There were corresponding versions for the unambiguous control sentences (d, e, f). Each sentence was typed individually without punctuation on a $3 \times 5$ in. card. (In this study we only used one of the possible nonambiguous interpretations as the control for each ambiguous sentence and three of each structural type for the surface structure and deep structure ambiguities. The decision as to which meaning of each lexical ambiguity to use as a control was arbitrary.)

14 (a) After taking the right turn at the 
Table 3

Mean Response Times (Seconds) for Ambiguous and Unambiguous Versions of Each Fragment Type in Lexical, Surface, and Underlying Structure Ambiguities (All Data from Experiment II)*

\begin{tabular}{|c|c|c|c|}
\hline & \multicolumn{3}{|c|}{ Fragment Type } \\
\hline & $\begin{array}{c}\text { In } \\
\text { Clause }\end{array}$ & $\begin{array}{l}\text { End of } \\
\text { Clause }\end{array}$ & $\begin{array}{c}\text { Start } \\
\text { Next } \\
\text { Clause }\end{array}$ \\
\hline \multicolumn{4}{|l|}{ Lexical } \\
\hline Ambiguous & 5.44 & 4.03 & 4.30 \\
\hline \multirow[t]{3}{*}{ Unambiguous } & 5.20 & 4.20 & 4.61 \\
\hline & $F<1$ & $F<1$ & $F<1$ \\
\hline & $p>.05$ & $\mathrm{p}>.05$ & $p>.05$ \\
\hline \multicolumn{4}{|l|}{ Surface Structure } \\
\hline \multirow[t]{3}{*}{ Unambiguous } & 4.25 & $\begin{array}{l}4.29 \\
4.70\end{array}$ & 5.07 \\
\hline & $F=3.17$ & $\mathbf{F}<1$ & $F=1.52$ \\
\hline & $\mathrm{p}<.10$ & $\mathrm{p}>.05$ & $p>.05$ \\
\hline \multicolumn{4}{|c|}{ Underlying Structure } \\
\hline Ambiguous & 6.07 & 4.93 & 5.22 \\
\hline \multirow[t]{3}{*}{ Unambiguous } & 5.03 & 5.65 & 4.76 \\
\hline & $F=5.88$ & $F=3.40$ & $F<1$ \\
\hline & $\mathrm{p}<.025$ & $\mathrm{p}<.10$ & $\mathrm{p}>.05$ \\
\hline
\end{tabular}

$*^{*} d f=1,47$

(b) After taking the right turn at the intersection

(c) After taking the right turn at the intersection I

(d) After taking the left turn at the

(e) After taking the left turn at the intersection

(f) After taking the left turn at the intersection I

15 (a) After the paper presented carefully limited

(b) After the paper presented carefully limited analyses

(c) After the paper presented carefully limited analyses they

(d) After the paper presented very limited

(e) After the paper presented very limited analyses

(f) After the paper presented very limited analyses they

16 (a) After the agent claimed the shooting of the Indians was

(b) After the agent claimed the shooting of the Indians was dreadful

(c) After the agent claimed the shooting of the Indians was dreadful

(d) After the agent claimed the shooting at the Indians was

(e) After the agent claimed the shooting at the Indians was dreadful

(f) After the agent claimed the shooting at the Indians was dreadful he

Six experimental groups were arranged from these sentences such that each group contained one sentence of each type from the 18 different types of sentences (ambiguous vs unambiguous control by three types of ambiguity by three fragment lengths = 18 sentence types). A group of 11 similar sentences was used for warm-up and one at the end to reduce end-of-session effects.

\section{Subjects}

The Ss were 24 M.I.T. summer school students and 24 Columbia, Hunter, and New York University students, native speakers of English who volunteered for paid participation.

\section{Procedure}

A stack of cards was presented to $S$ face down; $S$ was instructed to turn over a card. read the sentence fragment silently, and then, as soon as possible, to read the fragment and a completion aloud. $S$ was instructed to make the completion as well formed and relevant to the sentence fragment as possible. After the experimental session. $\mathrm{E}$ and $\mathrm{S}$ went over $\mathrm{S}$ 's responses to the fragments. $S$ indicated on a 5-point scale an evaluation of the appropriateness of the original response to the sentence fragment. $S$ was also asked, for each ambiguous fragment, whether he or she noticed its ambiguity at the time when he or she first saw it. The few responses for which $S$ reported having noticed the ambiguity were excluded from further consideration The entire experimental session was recorded. Turning over the stimulus card triggered a tone (inaudible to $S$ ) which was recorded on the response tape. The tape was later played back using a voice switch to determine the interval from stimulus presentation to onset of response.

\section{Results}

Analyses were performed on scores for the interval between stimulus presentation and onset of verbalization. The mean time to think up a completion was about $5 \mathrm{sec}$. The mean response times for each condition are given in Table 3. For each of the ambiguity types (lexical, surface, and underlying), three orthogonal contrasts were computed within a Fragment Type by Ambiguity analysis of variance (Hayes, 1963). The three contrasts compared response times for ambiguous vs unambiguous versions in each of the three fragment types. ${ }^{2}$ Table 3 presents the results of the contrasts for lexical, surface, and underlying ambiguities. None of the contrasts is significant for the lexical or surface structure ambiguities. For the underlying structure ambiguities, however, ambiguous fragments in the incomplete clause condition have significantly longer thinking times than do their control fragments ( $p<$ .025 ). For ambiguous fragments in the complete clause condition, the results are reversed, but not significantly, such that ambiguous fragments have shorter times than do their controls $(p<.10)$. Note (see means in Table 3 ) that the same pattern of reversal of ambiguity effect from incomplete to complete fragment types may also be present for surface structure ambiguities, although the differences do not reach significance. (Reanalysis using speed scores yielded no change in the significance of the contrasts reported.)

These results might be affected by the fact that they include sentence completion responses that were not appropriate to the stimulus sentence-that is, on occasion, S's response was clearly inappropriate to either meaning of the stimulus sentence fragment. This was operationally checked by having Ss rate the relative appropriateness of their own responses on a 5-point scale. There was no difference in this subjective rating across the ambiguity types. When we consider only the two best-rated categories of responses, the pattern of results is the same, as shown in Table 4.

When Ss are performing most appropriately to the task in their own subsequent opinion, they show the effect of clause segmentation and ambiguity. It remains the case that the effects were due primarily to underlying structure ambiguities. (Because of the difference in cells, orthogonal contrasts were not 
advisable; a by-S Wilcoxon matched-pairs signed-ranks test showed that the in-clause break result was significant at $p<.025$ (one-tailed) for underlying structure ambiguities, while the other differences were not significant.

\section{Discussion}

These results indicate that for the sentence completion task, underlying structure ambiguity does increase processing difficulty if the stimulus fragment is itself an incomplete clause. This finding was predicted by the assumption that the clause is the main perceptual unit of speech, that listeners assign a single interpretation to each such unit, and that the meanings of underlying structure ambiguities are more distinct from each other perceptually than are the meanings in other ambiguity types. When an ambiguous fragment is broken off before the completion of either underlying structure, then the two partial interpretations are both present and involve added processing complexity if a task is interposed at that point. If the fragment is broken off after completion of the perceptual unit, the perceptual unit is recoded with one interpretation and the fact that the unit was originally ambiguous does not affect the complexity revealed in later processing. When both interpretations are possible responses to the task, the processing time should be relatively fast in underlying structure ambiguities, since the two meanings utilize relatively independent perceptual rules. This pattern of results was found in Experiments I and II.

A number of issues remain concerning the general perceptual model. First, our stimuli did not critically test whether the perceptual unit relevant for our effects is the surface structure clause or underlying structure sentoid: in our materials, the complete units were simultaneously surface and underlying structure clauses [cf. (14b), (15b), and (16b)]. To test the role of underlying structure organization as the unit relevant to ambiguity, one would have to study the effects of fragments that are complete and incomplete underlying structure units without any surface structure clause markers. For example, the ambiguity in neither (17a) nor (17b) is in a complete surface structure clause, but (17a) completes an underlying structure unit [see Bever et al (1969) for a description of the relevance of such units in perceptual segmentation, using unambiguous sentence pairs like (17a) and (17b)]. If the underlying structure unit alone is sufficient for closure, the ambiguity in (17b) but not in (17a) should involve relative processing complexity.

17 (a) The English teacher's wife desired ...

(b) The English teacher's wife defied ...

Previous research has tended to refer to the structural definition of the ambiguity type as the main variable per se. However, our interpretation of the previously and
Table 4

Mean Response Times (Seconds) for Ambiguous and Unambiguous Versions of Each Fragment Type in Lexical, Surface, and Underlying Structure Ambiguity (Data from Best Two Subjective Rating Categories from Experiment II)

\begin{tabular}{|c|c|c|c|}
\hline & \multicolumn{3}{|c|}{ Fragment Type } \\
\hline & $\begin{array}{c}\text { In } \\
\text { Clause }\end{array}$ & $\begin{array}{l}\text { End of } \\
\text { Clause }\end{array}$ & $\begin{array}{c}\text { Start } \\
\text { Next } \\
\text { Clause }\end{array}$ \\
\hline \multicolumn{4}{|l|}{ Lexical } \\
\hline Ambiguous & 4.73 & 3.71 & 4.17 \\
\hline Unambiguous & 5.02 & 3.80 & 4.38 \\
\hline \multicolumn{4}{|l|}{ Surface Structure } \\
\hline Ambiguous & 4.37 & 4.51 & 3.72 \\
\hline Unambiguous & 4.38 & 3.71 & 4.52 \\
\hline \multicolumn{4}{|c|}{ Underlying Structure } \\
\hline Ambiguous & 5.79 & 4.50 & 4.53 \\
\hline Unambiguous & 4.42 & 4.75 & 4.59 \\
\hline
\end{tabular}

presently observed results classifies ambiguities in terms of the independence of the perceptual mapping rules used in the comprehension of the two interpretations. The hypothesis that it is the perceptual independence of interpretations that governs behavioral differences in response to ambiguities could be tested by using ambiguities of the same structural "type" that vary the relative differences of the perceptual rules used in interpreting each of the meanings. For example, (18a) and (18b) are both classified as "surface structure ambiguities," but the two underlying structures for (18b) differ more than those in (18a).

18 (a) John looked up the street

(b) They fed her dog biscuits

Similarly, (19a) and (19b) are both "underlying structure ambiguities," but the two underlying structures for (19b) differ more than those of (19a).

19 (a) The natives dislike sailing in the harbor

(b) The duck is ready to eat

The relative complexity of underlying structures awaits development of a serious formal metric. Finally, the paradigms we have used are not ideal tests of perceptual systems. The outcome is confounded with other response systems; the long response times attest to this. Within these limits, the experimental facts reported in this paper support our theoretical resolution of the apparent contradictions among previous findings on sentential ambiguity. Both the previous and presently reported results support the general theory of sentence perception outlined in the introduction which emphasizes the processes of clause segmentation, recoding clause by clause, and the use of direct mapping rules to assign internal relations to surface sequences.

\section{REFERENCES}

Abrams, K. Subject task and speech processing. Unpublished 
doctoral dissertation. University of Michigan. Ann Arbor. 1973

Abrams, K.. \& Bever. T. G. Syntactic structure modifies attention during speech perception and recognition. Quarterly' Journal of Experimental Psychology, 1969, 21, 280-290.

Bever, T. G. A survey of some recent work in psycholinguistics. In Specification and utilization of a transformational grammar. Scientific Report No. 3. Yorktown Heights, N.Y: IBM Corp, 1968. [Air Force Contract No. Af 19 (628), July 1968].

Bever, T. G. The cognitive basis for linguistic structures. In R. Hayes (Ed.), Cognition and the development of language. New York: Wiley, 1970.

Bever, T. G. Serial position and response biases do not account for the effect of syntactic structure on the location of clicks. Journal of Psycholinguistic Research, in press.

Bever, T. G., Kirk, R., \& Lackner, J. The underlying structure of sentences are the primary units of speech perception. Perception \& Psychophysics, 1969, 5, 225-234.

Bever, T. G., Lackner, J. R., \& Stolz, W. Transitional probability is not a general mechanism for the segmentation of speech. Journal of Experimental Psychology, 1969, 79, 387-394.

Cairns, $H$. The effects of bias on the processing and reprocessing of lexically ambiguous sentences. Journal of Experimental Psychology, in press.

Caplan, D. Clause boundaries and recognition latencies for words in sentences. Perception \& Psychophysics, 1972, 12, 73-76.

Carey, P., Mehler, J., \& Bever, T. G. Judging the veracity of ambiguous sentences. Journal of Verbal Learning \& Verbal Behavior, 1970, 9, 243-254

Chapin, P. G., Smith, T. S., \& Abrahamson, A. A. Two factors in perceptual segmentation in speech. Journal of Verbal Learning \& Verbal Behavior, 1972, 11, 164-173.

Fodor, J., Bever, T. G. The psychological reality of linguistic segments. Journal of Verbal Learning \& Verbal Behavior, 1965, 4, 414-421.

Fodor, J., Bever, T. G., \& Garrett, M. The psychology of language: An introduction to psycholinguistics and generative grammar. New York: MoGraw-Hill, in press.

Fodor, J., \& Garrett, M. Some syntactic determinants of sentential complexity. Perception \& Psychophysics, 1967, 2, 289-296.

Fodor, J., Garrett, M., \& Bever, T. G. Some syntactic determinants of sentential complexity, II: Verb structure. Perception \& Psychophysics, 1968, 3, 453-461.

Foss, D. Some effects of ambiguity upon sentence comprehension. Journal of Verbal Learning \& Verbal Behavior, 1970, 9, 699-706.

Foss, D., Bever, T. G., \& Silver, M. The comprehension and verification of ambiguous sentences. Perception \& Psychophysics, 1968, 4, 304-306.

Garrett, M. Syntactic structures and judgements of auditory events: A study of the perception of extraneous noise in sentences. Unpublished doctoral dissertation, University of Illinois, Urbana, 1965.

Garrett, M. Does ambiguity complicate the perception of sentences? In G. Flores d'Arcais and W. Levelt (Eds.), Advances in psycholinguistics. Amsterdam: North-Holland, 1970.

Garrett, M., Bever, T., \& Fodor, J. The active use of grammar in speech perception. Perception \& Psychophysics, 1966, 1, 30-32.
Hakes. D. Doss verb strusture effect sentence comprohencion? Perception \& Psychophysics. 1971, 10. 229-232.

Hakes. D.. \& Caims. H. Sentence comprehension and relative pronouns. Perception \& Psychophysics, 1970. 8. 5-8.

Hayes. W. Statistics for psichologists. New York: Holt. Rimchart \& Winston. 1963.

Holmes. V.. \& Forster. K. Click location and syntactic structure Perception \& Psychophysics, 1972. 12. 9-15.

Jarvella. R. I. Effects of sytax on running menory span for connected discourse. Psychonomic Science, 1970, 19. 235-236.

Jarvella, R. J.. \& Herman, S. J. Clause structure of sentences and speech processing. Perception \& Psychophysics, 1972. 11. 381-384.

Lackner, J., \& Garrett, M. Resolving ambiguity: Effects of biasing context in the unattended ear. Cognition. 1972. 4, in press.

Mackay, D. To end ambiguous sentences. Perception \& Psychophysics, 1966, 1, 426-436.

MacKay, D., \& Bever, T. G. In search of ambiguity. Perception \& Psychophysics, 1967, 2, 193-200.

Mistler-Lachman, J. L. Levels of comprehension in processing of normal and ambiguous sentences. Journal of Verbal Learning \& Verbal Behavior, 1972, 11, 614-623.

Seitz, M. AER and the perception of speech. Unpublished doctoral dissertation, University of Washington, 1972.

Smith, E. E. Choice reaction time: An analysis of the major theoretical positions. Psychological Bulletin, 1968, 69, $77-110$.

Valian, V. Talking and listening and responding. Unpublished doctoral dissertation, Northeastern University, 1971.

Wingfield, A., \& Klein, J. Syntactic structure and acoustic pattern in speech perception. Perception \& Psychophysics, $1970,9,23-28$.

\section{NOTES}

1. In this discussion we are not concerned with the distinction between underlying structure sentoids and surface structure clauses. The data are unclear as to the effect of underlving structure segmentation alone on click location (cf. Bever, Kirk, \& Lackner, 1969; Chapin et al, 1972).

2. Note that there is no uniform prediction for either effect of ambiguity or fragment type; the test of our hypothesis resides in the interaction and hence, the orthogonal contrasts. The Ambiguity by Fragment Type interactions had F ratios of .29 $(\mathrm{df}=2,94)$ for lexical, $2.78(\mathrm{df}=2,94)$ for surface, and 4.54 (df $=2,94)$ for underlying ambiguities. None of the $F$ ratios for fragment or ambiguity effects reached significance except for that of fragment type in the lexical ambiguities $(F=4.23$, $\mathrm{df}=$ 2,47 ). That effect was attributable to slower response times for all the incomplete lexical fragments.

(Received for publication October 28, 1972; revision received February $6,1973$. 